\title{
An Agent-based Cognitive Approach for Healthcare Process Management
}

\author{
Bian $\mathrm{Wu}^{1}$, Minhong Wang ${ }^{1}$, Hongmin Yun ${ }^{2}$, Haijing Jiang ${ }^{1,3}$ \\ ${ }^{1}$ Faculty of Education, The University of Hong Kong \\ ${ }^{2}$ Ophthalmological Department, Shengzhen Hospital, Beijing University \\ ${ }^{3}$ Faculty of Science, Nanjing University of Aeronautics and Astronautics \\ \{covenwu,magwang\}@hku.hk,hongminyun@yahoo.com,h_j_jiang@hotmail.com
}

\begin{abstract}
Healthcare organizations are facing the challenge of delivering high-quality services through effective process management. There have been frequent changes of clinical processes and increased interactions between different functional units. To facilitate the dynamic and interactive processes in healthcare organizations, an agent-based cognitive approach is presented in this study. The emphasis is placed on dynamic clinical and administrative process management, and knowledge building as the foundation for process management. The treatment of primary open angle glaucoma is used as an example to demonstrate the effectiveness of approach for healthcare process management.
\end{abstract}

Keywords-Software agent; process management; healthcare

\section{INTRODUCTION}

In healthcare organizations, there are a variety of processes, such as hospital administration by managers, registration for treatment by patients, test report generation by technicians, and diagnosis decision making by doctors. There is a trend to view healthcare in a multidisciplinary perspective, as numerous interactions and cooperation take place across different functional units in terms of information sharing, consultation, and combined treatments. These interactive and collaborative activities should be well organized and managed, without which the efficiency of healthcare service cannot be easily guaranteed. To meet these requirements, a process-oriented and cooperation-supported healthcare system is needed, especially with computer and information technology support [19].

To facilitate healthcare process management, workflow technology has been applied in a number of studies [14]. Workflow is defined as a process managed by a computer program that assigns the work, passes it on, and tracks its progress [1]. The main advantage of using workflow technique is to help establish, maintain, modify or even reconstruct business processes in various organizations. In the recent years, organizational environments have been changing from stable-and-closed to dynamic-and-open. Business processes are

\footnotetext{
Proc. 8th IEEE Int. Gonf. on Gognitive Informatics (ICHIO9) G. Baciu, Y. Wang, Y.Y. Yao, W. Kinsner, K. Chan \& LA. Zadeh (Eds.) 978-1-4244-4642-1/09/\$25.00 @2009 IEFE
}

becoming increasingly complex and dynamic as they seek to cope with a wide range of internal and external interactions and changes. Traditional workflow technologies for process management are often inadequate for complex and dynamic situations due to the lack of flexibility and adaptability [17]. This change has also happened in the healthcare sector. Due to newly discovered symptoms of a specific disease or need of further diagnosis, pre-planned clinical schedules always request adjustment; ad hoc changes of medical process are needed during execution. Besides, to fulfill a simple task such as gonioscopy for glaucoma diagnosis, cooperation among different functional units is required. In sum, frequent changes of clinical pathways and increased interactions between different units have become a big challenge in healthcare process management.

To develop a computer-based system that enables effective process management in healthcare, an agent-based cognitive approach is presented in this study. The term "agent" refers to a piece of software that can perceive its environment through its sensors and can act upon that environment through the effectors [16]. A healthcare management unit has similar characteristics with software agent, which has a set of goals (e.g., treatment effects and costs), perceptions of the environment (e.g., symptoms and records), and actions to take (e.g., recommendations for test or treatment, and inquiry). The highly dynamic and unpredictable nature of organizational processes makes agent-based approaches appealing. Using agent-based technology, we may decompose a complex process into a number of loosely coupled tasks and delegate the tasks to a number of software agents, each of which works autonomously and collaboratively in performing tasks and managing the whole process [17].

In this study, an agent-based and process-oriented healthcare system is developed. The system consists of three layers, the agent layer for healthcare process management, the database layer for maintenance of medical records and knowledge, and the interface layer for human-computer interaction. The emphasis is placed on dynamic clinical and administrative process management, and knowledge building as the foundation for process management. This agent-based and process-oriented healthcare system distinguishes from other 
healthcare systems in terms of continuous awareness of the healthcare environment, real-time dynamic decision making of healthcare processes, and rule-based knowledge engineering. The treatment of primary open angle glaucoma (POAG) is used as an example to demonstrate the effectiveness of this approach.

\section{RELATED WORK}

As an interdisciplinary field, the design and development of clinical or healthcare systems have received increased attentions. A number of studies have been conducted, such as integrated healthcare enterprise frameworks, computer-interpreted guideline implementation, and workflow-supported healthcare management $[14,3,18,8]$. Wirsz suggests an integrated healthcare platform be formulized via combining mostly heterogeneous application systems of different functional units [20]. Lenz and Reichert identify and distinguish organizational processes and medical treatment processes, and investigate how advanced process management technology can improve IT support for healthcare processes [10]. The workflow technique for process management aims to separate the flow logic from the application code. The adoption of workflow technique in business applications seems promising, especially for end users to design specific processes to facilitate their work $[12,11]$. However, most workflow systems often appear to be static and lack of flexibility to offer run time changes [4]. Due to the frequent changes and interactions in healthcare administration and clinical pathways during treatments, there is an urgent need to manage dynamic and collaborative processes in healthcare environment.

\section{SYSTEM ARCHITECTURE}

To meet the requirements arising from dynamic processes and cooperative functions in healthcare, an agent-based healthcare process management system is presented with a three-layer architecture.

\section{A. Agent Layer}

In the system, a group of software agents are proposed to perform healthcare tasks. Various interactions take place between the autonomous agents or between the agents and human users including doctors, nurses, technicians, and administrators (see Fig. 1). The design of software agents is process-oriented, i.e., agents are able to execute tasks according to process rules. What's more, process rules can be edited in a graphical form, which makes it easy to specify and update the rules. The details of each agent are elaborated as follows.

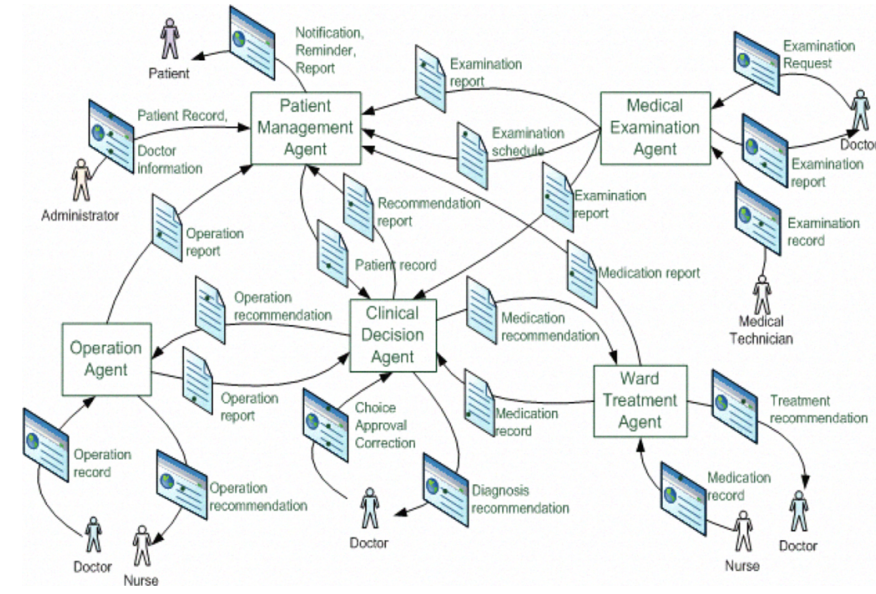

Fig.1. Multi-Agent and Human-Agent Communication

Clinical Decision Agent (CDA) works with doctors. It consists of three components: a knowledge base that captures glaucoma knowledge into a set of rules for clinical diagnosis decision making; a reasoning engine that generates diagnosis decision or treatment recommendations based on patient records and test results; and an interpreter that interprets the decisions or recommendations generated by the computer.

Patient Management Agent (PMA) works with clinical administrators and nurses, managing patient records and coordinating treatment processes. It also interacts with other agents for communication of patient records, test reports, and treatment records throughout the healthcare process.

Medical Examination Agent (MEA) works with doctors, medical technicians, or nurses, as well as communicates with PMA and CDA throughout the medical examination process. The medical examination process may go through several steps including patient records access, test scheduling, notification and reminder, and finally test report generation and delivery.

Ward Treatment Agent (WTA) works with doctors and nurses, as well as communicates with PMA or CDA during the inpatient treatment process. The activities include treatment scheduling, notification and reminder, information inquiry, and treatment report generation and delivery. The agent also maintains the information of treatment activities, progress, and patient states during the treatment process.

Operation Agent (OA) works with doctors and nurses, as well as communicates with PMA or CDA to manage the operation process. The process may go through several steps including patient records retrieval, operation scheduling, notification and reminder, and operation report generation and delivery. 


\section{B. Database Stage}

Medical information is stored in a database to support the healthcare functions. The information includes patient records, treatment records, examination records, diagnostic knowledge, and treatment knowledge. Considering information security and maintenance issue, different agents are associated with different data, with different rights to access or update the data. For example, PMA has the right to access patient records; MEA maintains the examination reports of all patients; OA keeps the operation records; and WTA reserves the medication records. The patient records, examination reports, operation records, and medication records can be acquired via communication with PMA, MEA, OA, and WTA respectively. Differing from data records, knowledge for clinical and administrative process management is more complicated; relevant techniques and tools are used to capture and model the knowledge into a set of rules or guidelines as discussed in section IV and V.

\section{Interface Layer}

This layer supports various interactions between human users (administrators, doctors, nurses, and medical technicians) and the software agents. Different type of user may access and interact with the system or software agents through relevant interfaces. An ophthalmologist may interact with a CDA, which is able to assist in diagnosis and treatment processes by providing decision making support based on patient basic information, diagnosis and medical status, test results, medical prescription, surgical procedure, and so on. A MEA helps a medical technician to manage test activities and reports, as well as to communicate with PMA, CDA, and a doctor regarding test arrangement and result report.

\section{COGNITIVE APPROACH FOR PROCESS MANAGEMENT}

To facilitate dynamic and interactive clinical processes, we need to capture a large amount of information and knowledge as the foundation for process management. In addition to clinical information such as patient records and treatment data, it is crucial to identify or set up the rules or guidelines as the knowledge for diagnosis and treatment decision making and clinical administration. To capture and represent the knowledge in the system, ontology-based technology is adopted in this study. Ontology is a way of knowledge engineering in computer programs. It is used to describe a set of concepts within a domain and the relationships between the concepts. Ontology provides a structured vocabulary with computerized specification of the vocabulary to model a domain. Using ontology, the narrative paper based guidelines or rules are encoded into computerized specifications.

To build the ontology, we use Protégé $[13,15]$ as the ontology representation tool. Protégé is a free and open-source platform that provides a suite of tools to construct domain models and knowledge-based applications with ontology. Protégé can be customized to provide domain-friendly support for creating knowledge models and entering data. Protégé can be further extended by way of a plug-in architecture and a Java-based Application Programming Interface (API) for building knowledge-based applications. In developing the proposed system, we use Protégé for knowledge building by way of class modeling, goal and criteria building, clinical algorithm construction.

In this study, we choose glaucoma treatment as an example to demonstrate the proposed approach. Glaucoma is a leading cause of blindness in most countries; the irreparable loss of vision by glaucoma requires lifelong healthcare. Meanwhile, the disease of glaucoma is well investigated; the process of the treatment is documented as various kinds of guidelines that can be used as reference for knowledge building in this case. There are many types of glaucoma, from which we choose the primary open angle glaucoma (POAG) for illustration.

\section{A. Knowledge Modeling}

We model the rules or guidelines for clinical decision-making into clinical algorithms (see Fig. 2). Based on the clinical algorithms, recommendations such as messages, drugs, and referrals can be generated for diagnosis, treatment, and other clinical process.

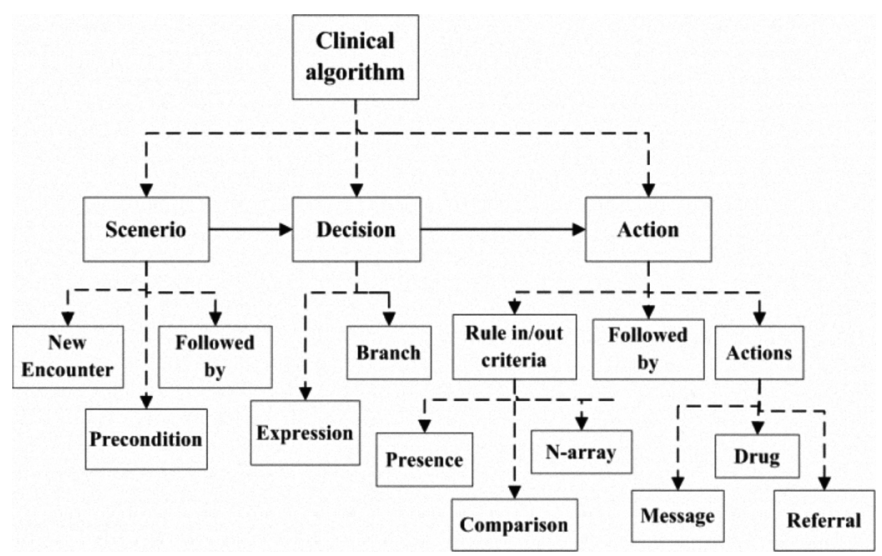

Fig.2. Hierarchy of clinical algorithm ontology

Each algorithm includes three nodes: scenario, decision, and action. Scenarios are mutually exclusive entry points for clinical algorithms, which exhibit unambiguous definition of patient states. A scenario contains several major properties called slots: a) the new encounter slot is used to enable/disable 
this scenario as a starting point; $b$ ) the precondition slot is to qualify the scenario as a starting point; and c) the followed by slot is a next step in the algorithm. A decision node contains: a) a choice step (e.g., severe IOP1 decrease $<30 \%$ ) which may lead to more than one choice; or b) a case step which has an evaluated expression to enable the execution of the next step node. An action is a node with a rule in/out criteria slot (e.g., if risk level $=$ severe and IOP decrease $>=30 \%$ ) and a defined action slot (e.g., Filtration surgery) in forms of message, drug recommendations, or referrals.

\section{B. Clinical Process Management}

The diagnosis and treatment process of glaucoma can be divided into several steps: 1) differential diagnosis; 2) further examination for establishing a baseline of intraocular pressure (IOP); 3) setting up a goal of intraocular pressure (IOP); 4) initial treatment; and 5) follow-up treatment.

\section{Differential Diagnosis}

In diagnosis period, comprehensive eye examination should be carried out, which includes: slit lamp biomicroscopy, Goldmann applanation tonometry (IOP), gonioscopy (to rule out angle closure, second causes of glaucoma), indirect ophthalmoscopy, and stereoscopic examination (for optic disc changes and retinal nerve fiber layer (RNFL) loss). After this, POAG or suspect can be differentiated via further examination. A part of the rules for differential diagnosis are listed below.

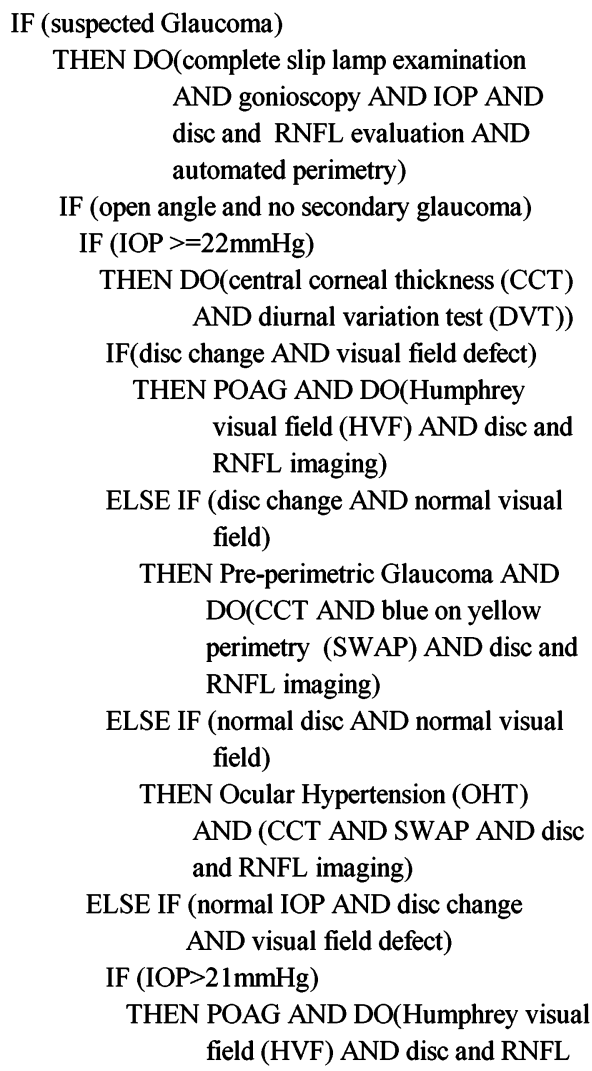

\author{
imaging) \\ ELSE Normal Tension Glaucoma (NTG) \\ AND DO(Humphrey visual field (HVF) \\ AND disc and RNFL imaging)
}

\section{Examination for Baseline Establishment}

Once POAG is determined, further examinations are needed for baseline establishment and initial treatment design. These examinations include at least 24-h diurnal diviation test (DVT) and central corneal thickness (CCT) test. In the examination, visual field loss can be classified into three categories:

IF ( $A<-6 \mathrm{~dB})$ and $(B<18$ points) and $(C<10$ points $)$

and $(\mathrm{D}<15 \mathrm{~dB})$

THEN Early defect

ELSE IF $(A<-12 d B)$ and $(B<37$ points $)$ and

$(C<20$ points $)$ and $(\mathrm{D}<0 \mathrm{~dB})$

THEN Moderate defect

ELSE IF $(A>-12 d B)$ and $(B>37$ points $)$ and

$(C>20$ points $)$ and $(D=0 \mathrm{~dB})$

THEN Severe defect

ELSE Good condition

where $\mathrm{A}$ indicates mean deviation index (MD); $\mathrm{B}$ and $\mathrm{C}$ indicate point number depressed below the $5 \%$ or $1 \%$ level on the pattern deviation plot, respectively; and $\mathrm{D}$ indicates the sensitivity of no point in the central $5^{\circ}$.

\section{Goal Setup}

After establishing a good baseline, it is obliged to set up a reasonable goal. The formula for target IOP rule is based on the patient's reference IOP (i.e., the mean of six separate IOP measurements taken in the course of two baseline visits) and reference visual field score (i.e., the mean of at least 2 visual fields taken during the two baseline visits). Below is the formula is used by the Collaborative Initial Glaucoma Treatment Study (CIGTS).

$$
\begin{aligned}
& \text { Target IOP }= \\
& \left(1-\frac{\text { Reference IOP+Visual Field Score }}{100}\right) \times \text { Reference IOP }
\end{aligned}
$$

where Visual Field Score ranges from 0 (no defect) to 20 (all points showing a defect at the $\mathrm{p}<0.005$ level).

\section{Initial Treatment}

Based on the goal established in STEP 3, an initial topical therapy is carried out to lower the pressure. The guidelines for the initial topical therapy are specified as follows:

Start the therapy in the worse eye first

IF (start topical therapy)

THEN choose first line medication

IF(met target IOP)

THEN continue and start in second eye

ELSE IF (not reduce IOP by at least $15 \%$ IOP or severe side effects)

THEN choose second line medication

ELSE IF(reduce more than $15 \%$ from baseline)

THEN reserve and try second line

IF (second target IOP)

THEN continue and start in second eye 
ELSE IF (second cannot met target IOP but have effect)

THEN try combination

ELSE step5

During the initial topical therapy, medications are involved. The rules for first line medication are listed below:

IF (cost-tolerable AND no inflammatory glaucoma)

THEN Prostaglandin analogues (PGA)

ELSE IF (target IOP=20\% IOP reduction AND no systemic beta-blocker AND no asthma AND heart block)

THEN $\beta$-blocker

ELSE IF (target IOP $>=30 \%$ )

IF (IOP reduction AND cost-tolerable AND no

inflammatory glaucoma)

THEN PGA

ELSE IF (no infants and MAO inhibitors users)

THEN $\beta$-blocker $+\alpha-2$ agonist

ELSE IF (no poor endothelial status)

THEN $\beta$-blocker+CAI

During the medications, the recommendation on dose usage is made according to the equations. Suppose drug A decreases outflow pressure by $\mathrm{pA}$ percentage, and drug $\mathrm{B}$ by $\mathrm{pB}$ percentage, respectively.

IOP2 = IOP1 - outflow pressure *pA

IOP3 = IOP2 - [IOP2 - (IOP1 - outflow pressure $\left.)^{*} \mathrm{pB}\right]$

where IOP1, IOP2, and IOP3 indicate IOP measured before drug $\mathrm{A}$, after drug $\mathrm{A}$ and before drug $\mathrm{B}$, and after drug $\mathrm{B}$, respectively. Based on (2), we can compare IOP2 and IOP goal to see if drug B should be used after drug A; based on (3), we may further compare IOP3 and IOP goal to estimate the topical therapy effect. Some studies such as $[7,5]$ provided a rough guide to the effect of various medications on the outflow pressure.

\section{Follow-up Treatment}

In the initial treatment of POAG, the first step topical therapy may not reach the initial intraocular pressure goal. Therefore, treatment algorithm after topical therapy [9] should be followed. Fig. 3 shows the modeling of this specific algorithm in Protégé. Pink circle indicates a scenario; yellow polygon indicates a decision; and green square indicates an action choice. They are linked by one direction arrow to show a follow-up relation with each other and form an algorithm chart. After implementation, the POAG treatment algorithm after topical therapy is represented as an instance of Management Algorithm Entity shown in Fig. 4. The pink circles, yellow polygons, and green squares in Fig. 3 are mapped to three subclasses (Scenario, Choice step, and Action Choice) of Management Algorithm Entity, respectively in Fig. 4.

The treatment in this step starts from the scenario that the IOP goal has not been reached after the initial topical therapy. First, the evaluation of the risk level is required, within which C/D ratio and field loss level examinations are executed to collect data for evaluation. Based on the evaluation result, a treatment pathway can be determined accordingly. There are three different pathways representing mild disease, moderate disease, and severe disease treatments. After the determination of the pathway, a further choice is made based on IOP threshold, which leads to different actions. For example, the mild level risk combined with less than $20 \%$ decrease in IOP should take laser action, and will lead to the follow-up phase scenario; the mild level risk with more than $20 \%$ decrease in IOP will lead to the follow up phase scenario directly. Similar processes are taken in treatment of moderate and severe level risks, but with more complexity in action choices like laser trabeculoplasty, additional drops, carbonic anhydrate inhibitor (CAI) therapy, or filtration surgery in different situations.

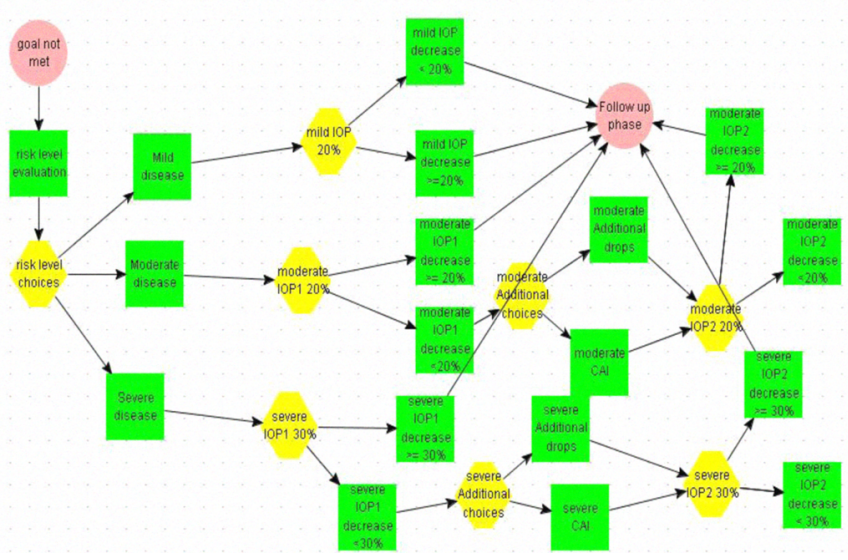

Fig.3. POAG treatment algorithm after topical therapy

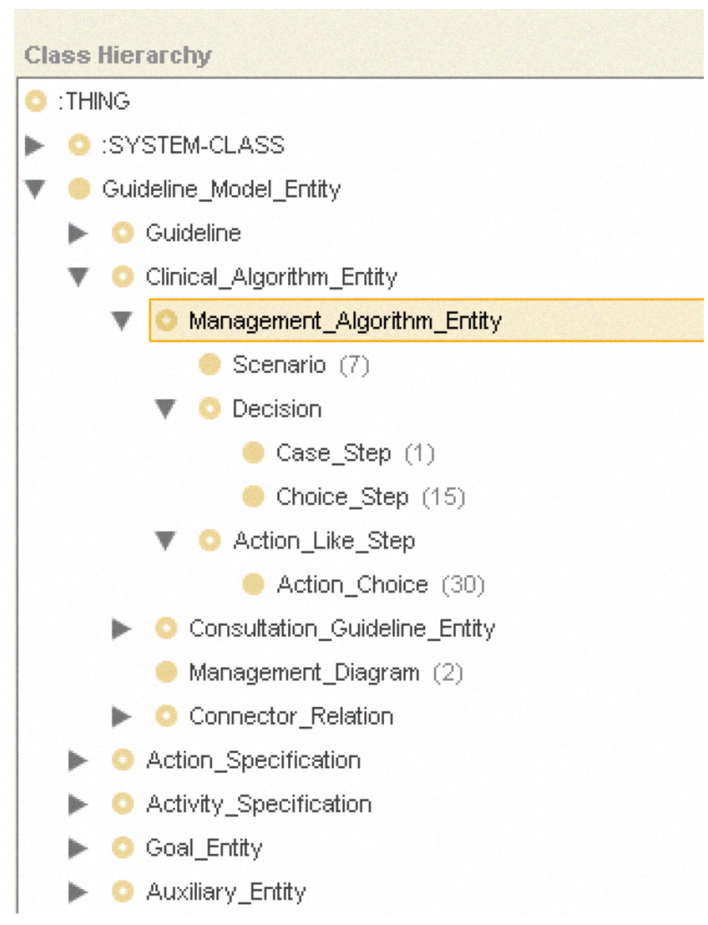

Fig.4. Class hierarchy in Protégé 


\section{IMPLEMENTATION}

In our system, five agents are developed, Patient Management Agent, Medical Examination Agent, Ward Treatment Agent, Operation Agent, and Clinical Decision Agent. For implementation of a multi-agent system, there are various choices of multi-agent platforms. JADE [6] is a widely used open source middleware developed by TILAB for the development of distributed multi-agent applications based on the peer-to-peer communication architecture. JADE is fully supportive of the Foundation for Intelligent Physical Agents (FIPA) specification. Messages exchanged by JADE agents have a format specified using the Agent Communication Language (ACL) defined by the FIPA international standard for agent interoperability.

In addition to multi-agent architecture, the implementation concerns the individual agent. After specifying clinical guidelines and process rules using Protégé, we use a plug-in called beangenerator, implemented by C.J. van Aart, to create the ontology definition class and the predicates, agent actions, and concepts classes [2] for implementation of individual agent. Agents can retrieve information and knowledge to perform tasks under control of rules or guidelines. Two types of rules are specified, one for clinical decision and another for administrative process control.

In our system, the agents are required to perform individual and collaborative tasks with other agents or human users throughout the healthcare process. The scenario of risk level evaluation, in the POAG treatment after topical therapy (see Fig. 3) is used as an example to outline the sequence of communication in the process. After the initial topical treatment fails to achieve the pressure goal, the rule-based POAG treatment algorithm triggers the next step of risk level evaluation, during which the Clinical Decision Agent may request the information about $\mathrm{C} / \mathrm{D}$ ratio and/or Field loss level for evaluation. The details of how the software agents and human users communicate during this process are outlined as follows.

As shown in Fig. 5, the sequence of communication includes a number of steps. After the doctor inputs the result of the initial topical therapy, the Clinical Decision Agent returns the advice of risk level evaluation due to the poor result of the initial topical treatment. After the doctor accepts the advice, Clinical Decision Agent requests additional data (e.g., C/D ratio) for risk level evaluation. Accordingly, the doctor sends the examination request to the Medical Examination Agent, and then some types of available test are suggested by the agent.
After the doctor selects an appropriate type of test, the medical technician may work with the Medical Examination Agent to settle the examination schedule and inform both the doctor and Patient Management Agent of the schedule. After the examination is completed, the technician makes the record of the result. With the help of Medical Examination Agent, the examination report is generated and delivered to the doctor and Patient Management Agent. Based on the report, the doctor may input relevant data to the Clinical Decision Agent and activate the risk evaluation process. The evaluation result is then generated by Clinical Decision Agent and reported to the doctor.

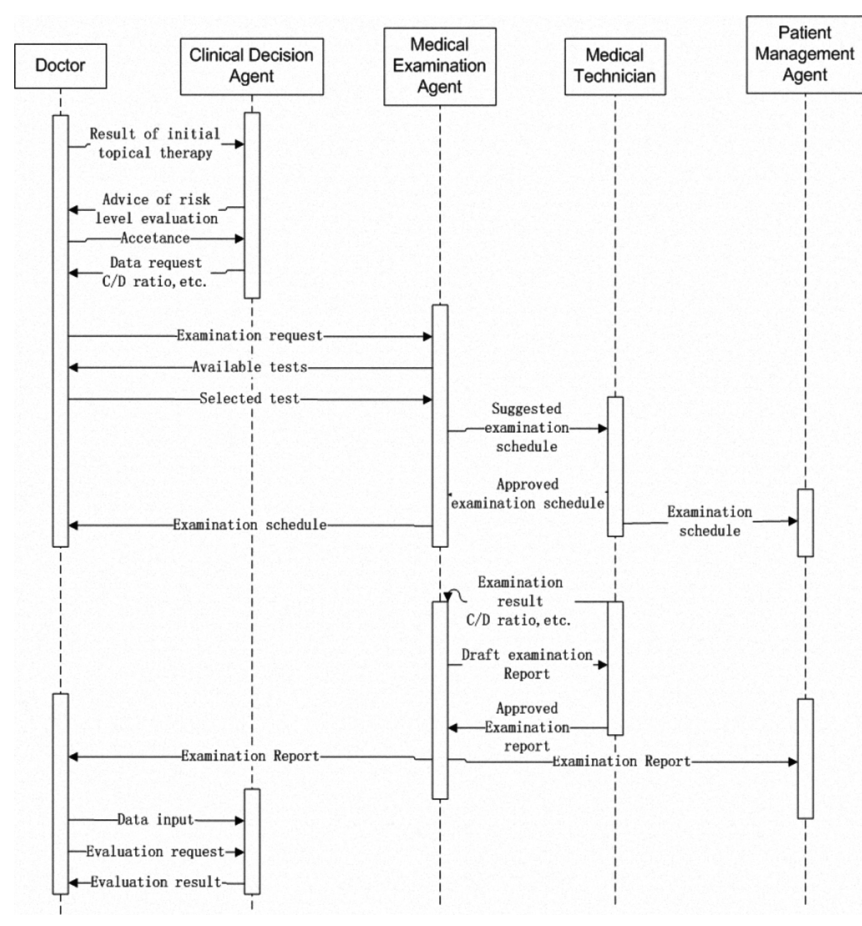

Fig.5. Sequence chart of communicative acts

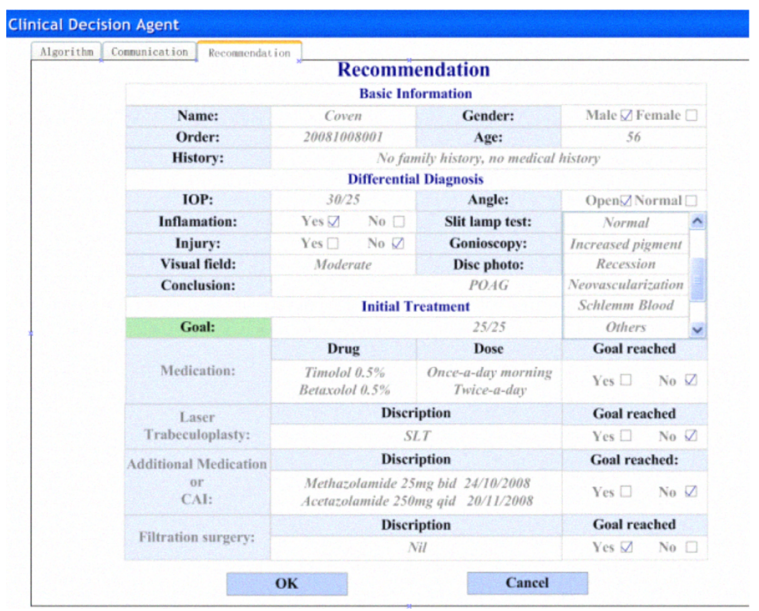


Fig.6. Profile of Recommendation

Fig. 6 depicts a screenshot of the prototype. The screen contains: a) basic information of a patient such as the name, gender, age, medical order, and history; b) diagnosis information on IOP test such as inflammation, injury, open angle, slip lamp test, goniosocopy, disc photo, and visual field test; and c) initial treatment information including medication records, laser therapy, CAI or additional treatment, and filtration surgery. Based on the information and relevant clinical guidelines, some recommendations are generate and presented.

\section{CONCLUSION AND FUTURE WORK}

This paper has presented a three-layer architecture of an agent-based healthcare process management system. The agent-based cognitive approach is applied to facilitate dynamic and interactive processes in healthcare management. The treatment of primary open angle glaucoma is used as an example for demonstration. After the implementation of the prototype, we will evaluate the effectiveness of the approach in terms of support for clinical and administrative process management in healthcare environment. Relevant clinical and administrative staff will be invited to use this system and give feedback and evaluation on the system.

\section{ACKNOWLEDGMENT}

This research is supported by UGC GRF Grant (716907) from the Hong Kong SAR Government and Seeding Funding for Basic Research (200711159052) from The University of Hong Kong.

\section{REFERENCES}

[1] AALST, W.M.P. \& HEE, K.M., Workflow management: models, methods, and systems, MIT press, Cambridge, MA, 2002

[2] CAIRE, G. and CABANILLAS, D., Jade Tutorial Application-defined content languages and ontologies, Manual, TILAB, 2004, available at http://jade.tilab.com/doc/CLOntoSupport.pdf

[3] CLERCQ, P.A.D., BLOM, J.A., Korsten, H.H. and Hasman, A., Approaches for creating computer-interpretable guidelines that facilitate decision support Artificial Intelligence in Medicine, 31 (1), 1-27, 2004

[4] DADAM, P., REICHERT, M., Kuhn, K., Clinical Workflows - The Killer Application for Process-oriented Information Systems? Proceedings of 4th International Conference on Business Information Systems, 2000

[5] DRANCE S.M. \& PA, N., The dose response of human intraocular pressure to pilocarpine. Can J Ophthalmol, 6-9, 1971

[6] BELLIFEMINE, F., CAIRE, G., GREENWOOD, D., Developing Multi-Agent Systems with JADE, Chichester, England; Hoboken, NJ: John Wiley, 2007

[7] Berson, F.G, Cohen, H.B., Foerster, R.J., Lass, J.H., \& Novack G.D., Levobunolol compared with timolol for the long-term control of elevated intraocular pressure. Arch Ophthalmol, 103 (3), 379-382, 1985.
[8] GOLDSTEIN, M. K., HOFFMAN, B. B., COLEMAN, R.W., et al., Implementing Clinical Practice Guidelines While Taking Account of Changing Evidence: ATHENA DSS, an Easily Modifiable Decision-Support System for Managing Hypertension in Primary Care, Proceedings of the AMIA Annual Symposium, 2000

[9] Hodapp, E.M.D., Parrish, R.G.M.D., Anderson, D.G., Clinical decision in glaucoma, Mosby-Year Book, Inc, 1993

[10] LENZ, R. \& REICHERT, M., IT support for healthcare processes- premises, challenges, perspectives. Data \& Knowledge Engineering, 61 (1), 39-58, 2007

[11] LEYMANN, F. \& ROLLER, D., Workflow-based Applications. IBM Systems Journal, 36, 102-123, 1997

[12] MEDINA-MORA, R., WINOGRAD, T., et al., The Action Workflow Approach to Workflow Management Technology, Proceedings of the Conference on Computer-Supported Cooperative. Work (CSCW), 1992

[13] O'CONNOR, M., SHANKAR, R., Tu, S., Nyulas, C., Das, A. \& Musen, M., Efficiently Querying Relational Databases Using OWL and SWRL The First International Conference on Web Reasoning and Rule Systems. Innsbruck, Austria, Springer, 2007

[14] REICHERT, M. \& DADAM, P., ADEPTflex - Supporting Dynamic Changes of Workflows Without Loosing Control. Journal of Intelligent Information Systems 10, 93-129, 1998

[15] RUBIN, D. L., NOY, N. F., MUSEN, M.A., Protégé: A Tool for Managing and Using Terminology in Radiology Applications. Journal of Digital Imaging, 20 (1),034-46, 2007

[16] RUSSELL, S. \& NORVIG, P., Artificial Intelligence: A Modern Approach, Upper Saddle River, N.J.: Prentice Hall, 2005

[17] WANG, M. \& WANG, H., From Process Logic to Business Logic - A Cognitive Approach to Business Process Management. Information \& Management, 43 (2), 179-193, 2006

[18] WEIN, B.B., EICHELBERG, M., IHLS, A., POISEAU, E., IHE "Integrating the Healthcare Enterprise"-an update for Information Technology Infrastructure for 2005. International Congress Series, 1281, 169-174, 2005

[19] WU, B., WANG, M., Yun, H., An Agent-Based System for Healthcare Process Management. Proceedings of International Conference on Enterprise Information Systems (ICEIS), Milan, 2009

[20] WIRSZ, N., Integrating the Healthcare Enterprise (IHE) achievements, expansion in new clinical domains and deployment. International Congress Series, 1281, 164-168, 2005 